\title{
तोi- \\ La relación de ayuda profesional, basada en la aceptación respetuosa del otro, como elemento facilitador del cambio
}

\author{
M. ${ }^{a}$ Petra Used Mínguez \\ DEPARTAMENTO DE PSICOLOGÍA Y SOCIOLOGÍA \\ UNIVERSIDAD DE ZARAGOZA \\ petri@unizar.es
}

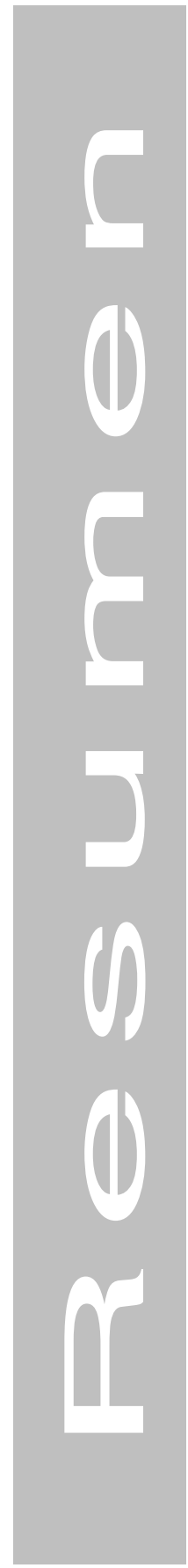

Resumen: Este artículo pretende aportar una reflexión sobre la importancia de la calidad en la relación establecida con los usuarios en los servicios de atención social especializada en intervención familiar.

Partiendo de la visión esperanzada en la sociedad que Concepción Arenal mantuvo a lo largo de su trayectoria y siendo conscientes de que, casi doscientos años después, su pensamiento, su sentido del ser humano y su legado humanista está presente en muchos profesionales del Trabajo Social, se intentará trasmitir algunos de los elementos que hacen referencia a la calidez en la relación de ayuda desde un actitud comprensiva con el dolor humano.

Palabras clave: Calidez, cambio, intervención familiar, relación. 


\title{
"The relation of professional help, based in the respectful acceptance of others, as a facilitator and change agent"
}

\begin{abstract}
This article aims to provide a reflection about the importance of the quality in the relationship between the users in the Family Intervention Specialized Social Care, stating that this is one of the facilitator and change agents.

Taking a hopeful view of the society as a starting point, which Concepción Arenal kept during her quiet experience and noticing that, almost after 200 years, her thoughts, her sense of human being and her humanist legacy is still alive in many professionals social workers; this article will try to show some of the agents that make reference to the warmth in the relation of help from an understanding attitude with the human pain.
\end{abstract}

Keywords: Warmth, change, family intervention, relationship. 


\section{La relación de ayuda profesional, basada en la aceptación respetuosa del otro, como elemento facilitador del cambio}

M. ${ }^{\mathrm{a}}$ Petra Used Mínguez

Recibido: 27-04-2012

Aceptado: 18-05-2012

\section{Introducción}

Múltiples son las cualidades de Concepción Arenal relacionadas con la cuestión social de su época, figura referencial para las generaciones venideras y persona luchadora por los derechos de la humanidad, su comprensión y sensibilidad ante el dolor humano es el punto de partida de todos sus estudios, que la convierten en impulsora de la dignidad del ser humano y de los derechos de los más desfavorecidos.

En su obra descubrimos elementos claros acerca de la persona del profesional que le sitúan en la relación de ayuda desde una actitud libre de generar resistencia en el ayudado, afirmando que la misma posición equivocada de superioridad se adquiere desde la mitificación que hacemos del saber técnico, frente al supuesto no saber de quien sufre la situación, acentuando con esta descripción su profundo conocimiento psicológico de la condición humana y predominando el respeto de la voluntad del pobre, que hasta en los casos más difíciles debe prevalecer por encima de todo. Enemiga de las injusticias, se siente comprometida en mejorar las condiciones de vida de los marginados, examinando al sujeto dentro de sí; es éste un rasgo que la sitúa "...en una corriente moderna puente entre la conquista del laicismo y las formuladas en principios universales comunes a las diferentes religiones". (Lacalzada, M.J. 1994 c: 17). 
Nos identificamos con la habilidad de esta gran reformadora social, que estimula con acierto la empatía de quien se dispone a dar ayuda, con su sensibilidad y buen criterio que se traduce en una intervención marcada por los límites del respeto hacia el ayudado, en definitiva por la humanidad hacia los más carenciados: "Moldear la actitud del que ayuda, para no errar en la acción de ayudar" (Arenal, 1863: 8 ), es su reivindicación a lo largo de su trayectoria y así queda reflejada en toda su obra.

Partiendo de la hipótesis "la calidad en la actuación precisa formular a los clientes los recursos más apropiados", no se debe obviar que en algunas situaciones estos clientes están obligados a acudir a un centro de tratamiento y sus defensas ante la posible intrusión en sus vidas las van a trasladar al profesional que les atiende, si bien es cierto que no todos los profesionales están preparados para encajar el reto de ayudar al que se siente obligado a que le ayuden, es necesario tener presente esta premisa, puesto que no es fácil en una primera fase la creación de una alianza que favorezca este desafío.

En la experiencia profesional de intervención familiar, que tomaré como referencia para desarrollar este artículo, puesto que es el contexto donde he ejercido la mayor parte de mi trayectoria laboral, se constata la importancia creciente de tener en cuenta al usuario y su familia: sus valores, costumbres, procedencia, cultura, composición etc..., estableciendo un contacto positivo y aceptando su propia narrativa, sin emitir juicios de valor que pueden determinar una relación inadecuada.

Una relación positiva facilita el proceso, contribuyendo a la mejoría. Por tanto, la eficacia no sólo depende de elegir la mejor técnica, y así lo avalan distintos autores (Frank, 1973; Grencavage y Norcross; 1990; Lambert, 1992), que han estudiado a fondo este aspecto de la intervención, afirmando en sus investigaciones que uno de los componentes fundamentales de esta recuperación son los aspectos de la relación.

Por supuesto, existen otros mecanismos (factores del cliente, expectativas, contexto) así como, un modelo teórico de guía y un diseño metodológico, siendo preciso observarlos para ofrecer calidad, eficacia y profesionalidad. 


\section{FACTORES DE LOS CLIENTES OBLIGADOS}

Denominamos clientes obligados a aquellos colectivos en los que la problemática está situada, de manera predominante, en la esfera interpersonal. Aquellas estructuras relacionales donde existen disfunciones entre dos o más miembros del núcleo convivencial y que presentan comportamientos desajustados, produciéndose un inadecuado funcionamiento o muestran dependencia crónica de las instituciones socio-sanitarias.

Se pueden considerar dentro de este apartado:

- Las familias multi-problemáticas generalmente se convierten en familias "multi-tratadas", lo cual a veces hace que se pierda una visión integral o completa del funcionamiento de la familia y de su capacidad de cambio.

- Las familias perciben, de forma más o menos consciente, que es muy común el desacuerdo y la falta de consenso entre los profesionales. Tampoco es infrecuente que las familias aprovechen o incluso promuevan la división entre profesionales cuando esta segmentación les confiere algún tipo de ventaja aparente.

Una de las preguntas que manifiestan los profesionales de la atención familiar es ¿cómo conseguir una buena relación con alguien que no ha acudido de forma voluntaria al servicio en el que se pretende ayudarles?, ¿y cómo lograr una buena relación con alguien que está irritado e incluso hostil por el hecho de tener que estar viendo a un técnico especializado en la intervención familiar?

En general, este tipo de intervención debería ser algo voluntario, pero hay ocasiones en las que la mediación de pareja y de familia incluyen esta situación paradójica; alguna de las personas que acude no quiere o incluso rechaza la propuesta.

Si bien es cierto que existen casos relativos a problemáticas inherentes a dinámicas familiares disfuncionales, junto con situaciones características de la propia dinámica familiar, también lo es que en la actualidad se ve más que nunca cómo en las familias la diversidad de tipologías, modelos educativos, estilos de relación y constitución de las mismas no facilitan el establecimiento de una relación positiva, siendo uno de los principales objetivos en la primera fase para el profesional que, 
en ocasiones "está obligado a atenderles" ya que, en un principio este trato se produce bajo el signo de un rechazo activo.

De esta forma, la evolución nos conduce a una población:

- Con un perfil más secundario, con una capacidad de demora y planificación que le sitúa en el tránsito casi consumado de lo disocial a lo antisocial.

- Derivada en un número cada vez mayor de instituciones públicas de protección que se ven desbordadas por situaciones y conflictos más violentos que en épocas anteriores. Su inclusión suele estar sometida a la interposición de denuncias, cuya reparación está supeditada a la asistencia de sesiones programadas con la familia y el agente infractor (en los casos de menores) o a programas de reinserción (en los casos de toxicomanías). Ello cobra especial importancia si estas figuras educativas constituyen el escenario representativo de la sociedad sobre el que el cliente desplaza su resentimiento contra las figuras parentales

- Estos perfiles descritos suelen conllevar una mayor disociación de los contenidos conflictivos y problemáticos de su historia personal, así como un aumento de la negación sobre los actos que han cometido y que son objeto de derivación a estos servicios de atención programada contribuyendo a ampliar la escenificación de un rechazo activo de índole social.

En el caso de familias que han sido calificadas como multiproblemáticas o multi-stressed (Nadser, 1999), usualmente familias de estrato social bajo y que reciben atención especial de múltiples servicios (servicios sociales, drogodependencias, educación familiar, medidas de protección infantil....), la situación de conflicto y desconfianza hacia los diferentes profesionales es ciertamente más frecuente y también potencialmente más negativa en relación con la consecución de una buena relación con el profesional ( Imber-Black, 1988; MacKinnon, 1998).

\section{LOS PROFESIONALES DE LA INTERVENCIÓN}

Atender personas que presentan dificultades requiere del profesional una ocupación permanente de sí mismo: la capaci- 
dad de integrar la dimensión profesional y la personal. De esta integración bidimensional resulta la persona-profesional, en la que se incluye, por un lado, el ámbito competente, en que encontramos todos aquellos requisitos que se exigen para la práctica de una función específica (técnicas teorías, códigos, etc.) y, por otro, el ámbito personal, en que se reúnen la disposición y las habilidades para un constante desarrollo.

Por otra parte, esta integración para desarrollar la profesión se ejerce mediante la autogestión de la persona. Ahora bien, hay que añadir que funcionar adecuadamente en el ámbito personal no es suficiente para funcionar bien en la vertiente profesional. Para llevar a cabo la autogestión es preciso integrar competencias personales y requisitos profesionales, y hacer encajar los objetivos con las capacidades de actuación. Con este propósito hay que tomar en consideración las preferencias y los sentimientos propios, de los que se debe saber hacer un uso adecuado, pues las normas y valores propios influyen en las acciones, y es preciso concienciarse de cuáles son las limitaciones y competencias en la acción.

En el sistema profesional también encontramos unos niveles de gratificación o de malestar inherentes a la práctica.

Cuando los sistemas de ayuda profesionalizada: el sistema de salud mental, educativa, y de servicios sociales de cualquier comunidad se desarrollan y se mejoran, esto se suele traducir en una mayor complejidad y especialización de los profesionales de ayuda. Resulta difícil para los usuarios establecer alianza con un sistema de múltiples profesionales que tiene visiones diferentes, responsabilidades e intereses distintos, horarios y procedimientos diversos, y cuya coordinación a veces requiere un tiempo que no coincide con el tiempo de las familia (Escudero V., 2006).

La mala relación suele ser una consecuencia de la desconfianza generalizada hacía los profesionales de ayuda.

Para un paciente, pareja o familia que son obligados por alguna instancia externa a acudir a un servicio de atención especializada y muestran una actitud de "espectadores" o expresan hostilidad y resentimiento, el técnico que les atiende necesitaría saber el contexto de coerción que seguramente ha marcado y está marcando esta actitud de rechazo. 
Si el objetivo del profesional se focaliza únicamente en los problemas por los que la familia ha sido derivada (adicción, abandono, descuido, arbitrariedad, desinterés en las funciones educativas) y en la actitud no colaboradora o de rechazo que presentan inicialmente hacía él, probablemente la relación no va progresar y esa falta de colaboración podrá tomarse como "otra prueba más" de la disfuncionalidad o patología de los clientes. Sin embargo, cuando se analiza cómo han vivido los padres o familiares la intervención social (de los trabajadores de los servicios sociales, de protección, del sistema educativo, sanitario...) y las causas de la profunda desconfianza entre clientes y profesionales, se podrá entender que, inicialmente se muestren invadidos por un sentimiento de intrusión que dificultará la creación de una ambiente de seguridad y conexión emocional necesario para el afianzamiento de una relación funcional y positiva.

MacKinnon (1998) sugiere que si el técnico pone más atención a la influencia del género y la clase social, entre otras variables, será más capaz de ofrecer una ayuda experta que no esté marcada por la relación de poder entre profesionales y clientes. Ese aspecto es central para una comprensión de la dificultad de crear alianza con usuarios que se consideran "prisioneros" de la intervención.

Desde una perspectiva centrada en la creación de alianza con clientes que no colaboran o que muestran actitudes iniciales de rechazo, la consideración de su punto de vista y su experiencia previa sobre el contexto de ayuda -social o terapéuticaes el primer ejercicio que el profesional puede hacer para favorecer la alianza. Ese ejercicio suele propiciar una actitud más comprensiva y una dosis mayor de paciencia y motivación en el trabajo con clientes obligados o presionados. En nuestra experiencia, cuando se cuida esta posición continuamente respetuosa hacia las razones que motivan la desconfianza del cliente, es más fácil que se promuevan las primeras muestras de confianza. Habitualmente se formulan en expresiones o testimonios del tipo "éste/a es diferente" o "éste/a parece que realmente quiere ayudarnos". 


\section{UNA INTERVENCIÓN ORIENTADA A OBJETIVOS}

Además de unos criterios de inclusión, existen una serie de objetivos generales y específicos para el trabajo con las familias, en función de las características de la problemática por las que fueron derivadas.

Entre los objetivos de las intervenciones familiares, desde un modelo sistémico y en un contexto no clínico (social y judicial) apoyamos un trabajo de calidad y eficacia destacando aspectos como la calidez de la relación establecida con nuestros usuarios, para conseguir la aceptación de corresponsabilidad de los miembros familiares, potenciando los recursos internos de las familias y ayudando a su movilización para abordar las dificultades en una etapa del ciclo vital que está presentando disfuncionalidad en su funcionamiento relacional.

El acercamiento a la familia, está muy relacionado con la aceptación de la diversidad familiar actual. Aunque prevalece la familia nuclear, hay un incremento de otras formas familiares -familias monoparentales, familias de hecho, familias reconstituidas,...-. Más importante que el aumento de estas formas familiares es la aceptación mayoritaria de la diversidad familiar.

La calidez en la relación engloba la creación de espacios seguros, de participación y de comunicación alternativos para todos los miembros de la unidad familiar que acudan a las entrevistas. La confidencialidad es un aspecto fundamental en el proceso que refuerza la confianza del usuario en el profesional que le atiende.

\subsection{La calidad en el acercamiento con clientes obligados}

El trabajo con clientes obligados, como ya se ha comentado, tiene una doble dificultad de partida:

1. La que afecta a los profesionales, cuando hay unos objetivos de la intervención que están pautados por la institución responsable que atiende el caso y en ocasiones por los agentes derivantes, que hacen constar una definición previa de quiénes son los usuarios del tratamiento e incluso de la duración y frecuencia del proceso. La alianza 
entre profesionales implica compartir la responsabilidad y respetar el ámbito de trabajo de cada experto, es necesario disponer de unas condiciones adecuadas para establecer una relación de ayuda con el cliente, para ello se precisa que cada profesional realice adecuadamente su labor, coordinándose con otros técnicos, que forman parte de su equipo de trabajo.

2. Otra dificultad está relacionada con la familia. Se ha hecho referencia, a las situaciones en las que la obligación de acudir a un servicio especializado en intervención familiar no es una condición de obligado cumplimiento, pero existe una presión muy fuerte.

En algunos casos puede que acepten que necesitan ayuda o que precisan cambios en su vida, pero no han sido ellos los que han elegido este recurso. En otros casos ni siquiera hay un reconocimiento de la existencia de un problema por lo que la, denominada ayuda, quizá sea contemplada como otro elemento punitivo más de su proceso de vida.

En cualquier caso, tenemos que entender esos factores como inherentes a nuestro trabajo, intentando conseguir un compromiso, fomentando la adherencia y favoreciendo la confianza.

\subsection{Razones para crear coalición con clientes obligados o pre- sionados}

La mala relación podemos interpretarla como un "síntoma" del conflicto intra-familiar o inter-personal:

Cuando dentro de los miembros citados, que acuden a las sesiones pautadas, coincide una forma "solicitante" en un miembro de la familia y una actitud de "invitado" o "prisionero" en otro u otros, probablemente ambos tipos de relación sean una expresión del patrón relacional que les está impidiendo resolver el problema. Con frecuencia en este tipo de situación se están dando sentimientos de culpa, rechazo y temor a ser juzgados, por lo que el profesional necesitará establecer un espacio de seguridad para los diferentes miembros, haciéndoles ver que 
entiende sus puntos de vista y sus reacciones, al tiempo que les muestra respeto y comprensión hacia las distintas posiciones que cada uno describe, intentando que, desde la neutralidad todos y cada uno de los asistentes se sientan parte de un todo, únicos en sus diferencias y competentes para conseguir mejorar la situación-dificultad por la que están pasando.

Resulta necesario para la colaboración de los clientes obligados el establecimiento de una buena alianza con el profesional, proporcionando un contexto de seguridad que favorezca un inicio de colaboración, puesto que a través de ella se favorecerá el cumplimiento de objetivos que ayuden a reducir los síntomas objeto de la intervención.

Es preciso determinar con transparencia los compromisos y limitaciones que familia y profesional asumen como establecidas por la entidad que les ha puesto en contacto, cuando este trato proceda de una derivación "impuesta", siendo elemental la creación de alianza para el trabajo conjunto.

\subsection{La calidez en la negociación de objetivos}

Es bastante frecuente escuchar la impotencia y frustración que sienten los trabajadores de distintos servicios, cuyos usuarios corresponden a la cualidad de "clientes obligados" porque no colaboran con los objetivos impuestos.

Cualquier objetivo debe ser negociado con la familia y sobre todo trabajar con mucho tacto. La consecución de un nivel mínimo en la dimensión compartir el propósito (Escudero V., 2006) suele requerir de algún acuerdo de compromiso para, respetando las diferentes posiciones ante el problema, hacer algo en común por mejorar la situación.

La actitud de no imposición de objetivos, ni de tareas a los clientes sin un compromiso previo de colaboración entre ellos y con el experto es un criterio fundamental. El profesional debe negociar con delicadeza cada nuevo pequeño compromiso que adquiere con este tipo de usuario. Debe aprovechar la motivación del cliente demandante para colaborar en la búsqueda de soluciones que no reproduzcan pautas de culpabilidad y acusaciones. 
Desde una perspectiva centrada en la creación de alianza terapéutica con clientes que no colaboran o que muestran actitudes iniciales de rechazo, la consideración de su punto de vista y su experiencia previa sobre el contexto de ayuda -social o terapéutica- es el primer ejercicio que el técnico puede hacer para favorecer la coalición.

En nuestra experiencia, cuando un profesional tiene esta posición continuamente comprensiva hacia las razones que motivan la desconfianza del cliente, es más fácil que el usuario proporcione las primeras muestras de confianza.

\subsection{Las características de la relación profesional}

Tomando como reseña las aportaciones de Carl Rogers en relación con la psicoterapia humanística, y partiendo de la base que nos proporcionan algunas claves de su pensamiento que coinciden con la contribución que, desde este artículo se está desarrollando y con el objetivo de argumentar la relación entre el paciente y la persona del profesional, así como las características necesarias para establecer relaciones respetuosas desde una actitud poco directiva que faciliten el cambio, se puede afirmar, que una de la premisas de las que parte este gran humanista, es que si el profesional posee la capacidad de ponerse en el lugar del otro, concibiendo y apreciando el mundo como él lo concibe podrá acompañarle en su proceso, pues se trata de "percibir a través de los ojos del cliente". (Rogers, 1981, pág. 42).

Ya en la década de los 1950, Rogers refirió que los factores habituales de la relación terapéutica tenían tanta o más importancia que los componentes específicos de las distintas técnicas, surgiendo de esta premisa el estudio y profundización en la reciprocidad establecida dos elementos "relación-técnicas" y que dio como resultado la terapia centrada en le cliente, junto con "un abandono del interés por técnicas concretas que logren causar un cambio", y una "indagación de un estilo específico de relación que va a ser favorecedor del cambio" (Sánchez Rodríguez, 2000, pág. 101).

Rogers menciona el término "ayudar", en contraposición a "no enseñar", a la persona a aceptarse a sí mismo, conside- 
rando necesarias y suficientes tres características en la relación profesional para el cambio personal:

1. La empatía. Entendida como la habilidad del técnico de situarse en el lugar del usuario, siendo importante que esta empatía esté observada por él.

2. Aceptación positiva incondicional. Creo que esta característica no precisa demasiada justificación. El profesional acepta y respeta enteramente todo cuanto el usuario le comunica.

3. Autenticidad. Congruencia. Coherencia. Con esto Rogers se refiere a que la conducta del profesional ha de ser genuina, no simulada, propia y personal, no fingida; esto en ocasiones, implica poder expresar al cliente sentimientos experimentados por él en determinadas circunstancias. El cliente ha de verlo como persona coherente en lo que hace, lo que dice, y lo que siente, y para que así suceda, éste ha de serlo.

Se puede afirmar que los aspectos que se producen en el estilo de relación profesional-cliente y que están basados en "la empatía, la calidez, la confianza, la aceptación y la voluntad de correr riesgos" independientemente de la orientación metodológica y de las técnicas utilizadas, revelan un tanto por ciento elevado en los cambios efectivos sucedidos en el proceso de ayuda.

\section{CONCLUSIONES}

Se ha comentado la importancia de crear una "buena alianza con los usuarios", concepto cuyo significado podemos correlacionar con el establecimiento de una buena relación, expresión reconocida por su validez en los procedimientos de los distintos modelos de psicoterapia como componente especifico de colaboración en el tratamiento.

Queda detallada la necesidad de tener en cuenta la colaboración de los usuarios en las intervenciones, a modo de garantía, eficacia, calidad y satisfacción en el cumplimiento de los objetivos diseñados en el proceso, demostrando que la percepción que tienen los clientes de la figura del profesional es mejor 
predictor de los resultados que la que tiene el técnico que los atiende (Bachelor, 1991).

Los beneficios de una perspectiva orientada al cambio en la que se puntúa de manera imprescindible la calidez en la relación establecida, en la comprensión del individuo y de su trayectoria de vida, unido al respeto del dolor y sufrimiento de quiénes se encuentran en su trabajo en la situación de ayudar a otros, compromete a revisar perspectivas que, en determinadas ocasiones desvirtúan este compromiso que para Concepción Arenal era tarea primordial en su trayectoria de implicación con la injusticia y el sufrimiento humano.

No obstante este tipo de configuración que nos ayuda a reconocer las posibilidades del otro, amplia nuestro ejercicio profesional al orientarnos hacia el potencial que "el otro posee", pudiendo convertirse en una perspectiva para la vida, en cierta medida es "una forma de ser", puesto que creemos en lo que hacemos y lo ponemos en práctica en nuestra propia existencia.

De un modo más personal, la experiencia nos demuestra que en estos años de intervención en distintos programas "no clínicos" (rehabilitación de toxicómanos, grupos de mutua ayuda, intervención familiar con jóvenes..., atención a la diversidad) y tomando como referencia los cuestionarios de valoración final aplicados a las familias y usuarios, podemos concluir que las variables mas valoradas por estos colectivos han sido el buen trato recibido, por lo que seguimos apostando por una relación de calidad, donde prime el respeto, la empatía y la coherencia establecida con nuestros clientes.

\section{Referencias bibliográficas}

BACHELOR, A. (1991). Comparación y relación de diversas dimensiones en la relación y creación a alianza con clientes en terapia. Psychotherapy, 28, 534-549.

CAMPO ALANGE, M. (1973). Concepción Arenal 1820-1893: Estudio biográfico documental. Madrid: Revista de Occidente.

FISHMAN H. CH. Y ROSMAN B.L. (Comps) (1998). El cambio Familiar: Desarrollos de modelos. Buenos Aires: Gedisa.

FRANK, J. D. y FRANK, J. B. (1991). Persuasion and bealing: A 
Comparative estudy of psichotherapye ( $3^{\mathrm{a}}$ ed.). Baltimore: Johns Hopinkins University Press.

FRIEFLANDER, M. L., ESCUDERO, V. y HEATHERINGTON, L. (2006). Therapeutic alliance en couples and family theraphy. Washington: American Psychological Association.

IMBER-BLACK, E. (1988). Families and Larger Systems: $A$ Family Therapist's Guide Through the Labyrinth. New York: The Guilford Press

LACALZADA DE MATEO, M.J. (1994 a). Mentalidad y proyección social de Concepción Arenal. El Ferrol: Cámara Oficial de Comercio, Industria y Navegación.

LACALZADA DE MATEO, M.J. (1994 b). Concepción Arenal: Un perfil olvidado de mujer y de Humanista. Arenal: revista de historia de las mujeres, 30 (1), 71-102.

McKINNON, R. I. y PILL., H. (1998). International Overborrowing: A descomposition of Credit and Currency Risks. World Development, 26 (7), 1267-1282.

NAVARRO GÓNGORA J. (1992). Técnicas y programas en terapia familiar. Buenos Aires: Paidós.

PITTMAN, F. S. (1990). Momentos decisivos: Tratamiento de familias en situaciones de crisis. Barcelona: Paidós.

ROGERS, C. y ROSENBERG, C. (1981). La persona como centro. Barcelona: Herder.

SÁNCHEZ RODRÍGUEZ, V. (2000). La resistencia al cambio en psicoterapia: Una perspectiva constructivista. Tesis Doctoral no publicada. Universidad Autónoma de Madrid. 